\title{
Project manager selection by using fuzzy simple additive weighting method
}

\begin{abstract}
By implementing Fuzzy Simple Additive Weighting (SAW) method for selecting project manager in MAPNA Company a multi criteria decision making (MCDM) method was conducted in this paper as part of an extensive research. Project manager selection is another tough and complex issue among decision making problems. In order to use a systematic method, a framework is demonstrated in this paper to assist a group of decision makers to use linguistic variables for rating candidates. Identifying the best candidate among participants for project manager selection is the goal of this study. To achieve this objective, a fuzzy SAW method has been used. The validation of these models were inspected by using a case study of project manager selection in order to choose a right PM for project manager position in a project based company. The feedbacks reveal that this model is quite reliable in selecting project managers and can ameliorate the efficiency of decision making process.
\end{abstract}

Keyword: Fuzzy SAW; Linguistic variables; Project manager selection; Project based organization; Project management 